\title{
Efficient Robust Optimization of Metal Forming Processes using a Sequential Metamodel Based Strategy
}

\author{
J.H. Wiebenga*, G. Klaseboer ${ }^{\dagger}$ and A.H. van den Boogaard** \\ * Materials innovation institute (M2i), P.O. Box 5008, 2600 GA, Delft, The Netherlands \\ ${ }^{\dagger}$ Philips Consumer Lifestyle, P.O. Box 201, 9200 AE, Drachten, The Netherlands \\ ${ }^{* *}$ University of Twente, P.O. Box 217, 7500 AE, Enschede, The Netherlands
}

\begin{abstract}
.
The coupling of Finite Element (FE) simulations to mathematical optimization techniques has contributed significantly to product improvements and cost reductions in the metal forming industries. The next challenge is to bridge the gap between deterministic optimization techniques and the industrial need for robustness. This paper introduces a new and generally applicable structured methodology for modeling and solving robust optimization problems. Stochastic design variables or noise variables are taken into account explicitly in the optimization procedure. The metamodel-based strategy is combined with a sequential improvement algorithm to efficiently increase the accuracy of the objective function prediction. This is only done at regions of interest containing the optimal robust design. Application of the methodology to an industrial V-bending process resulted in valuable process insights and an improved robust process design. Moreover, a significant improvement of the robustness $(>2 \sigma)$ was obtained by minimizing the deteriorating effects of several noise variables. The robust optimization results demonstrate the general applicability of the robust optimization strategy and underline the importance of including uncertainty and robustness explicitly in the numerical optimization procedure.
\end{abstract}

Keywords: Metal Forming Processes, FEM, Optimization, Uncertainty, Robustness, Sequential Optimization

PACS: $02.60 . P n$

\section{INTRODUCTION}

When dealing with the numerical optimization of metal forming processes using computationally expensive FE simulations, arriving at an optimal process is only one side of the coin. The other challenge is to optimize towards robust metal forming processes. More specifically, the goal is to improve the quality of a product or process by minimizing the deteriorating effects of uncertain parameters. In fact, very often an obtained deterministic optimum lies at the boundary of one or more constraints that constitutes a sharp border between acceptable products and waste. Variations in material properties, lubrication or process settings will now lead to a product rejection rate of approximately $50 \%$ in practice.

To avoid such waste, uncertainty has to be taken into account explicitly in any numerical optimization strategy. Apart from control variables $(\mathbf{x})$, the process is influenced by stochastic variables or noise variables $(\mathbf{z})$; see the process-diagram in Figure 1(a). The input variation is subsequently translated to the response $(f)$ which will now also display a probability distribution instead of just a deterministic value. Process robustness is related to the amount of variation in the process response, i.e. the narrower the response distribution, the more robust the manufacturing process. Robustness is thus a measure of the reproducibility of products that result from applying the process. An overview of the most important developments in the field of robust optimization can be found in [1] and [2].

In this paper, a robust optimization strategy is proposed that bridges the gap between deterministic optimization techniques and the industrial need for robustness. In the first section of this paper, the strategy that has been developed will be introduced. The modeling step and sensitivity analysis will be discussed in detail in the second section, after which the robust optimization procedure is described in detail. The paper continues with the introduction of a sequential optimization algorithm. The final section demonstrates the applicability of the strategy through the robust optimization of an industrial V-bending process. 


\section{A ROBUST OPTIMIZATION STRATEGY}

The proposed robust optimization strategy consists of 10 steps. A flowchart is presented in Figure 1(b). This flowchart serves as a guide through the process of mathematically modeling and solving the robust optimization problem in a structured way. The strategy was specifically developed for solving optimization problems including time-consuming FE simulations and was implemented in the optimization software OPTFORM developed at the University of Twente [3]. The software is applicable to any metal forming process and is suitable for use in conjunction with commercially available FE packages.

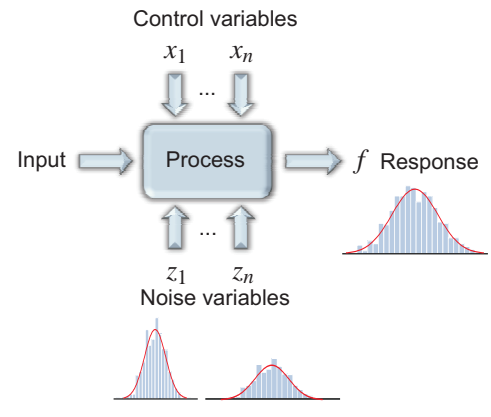

(a)

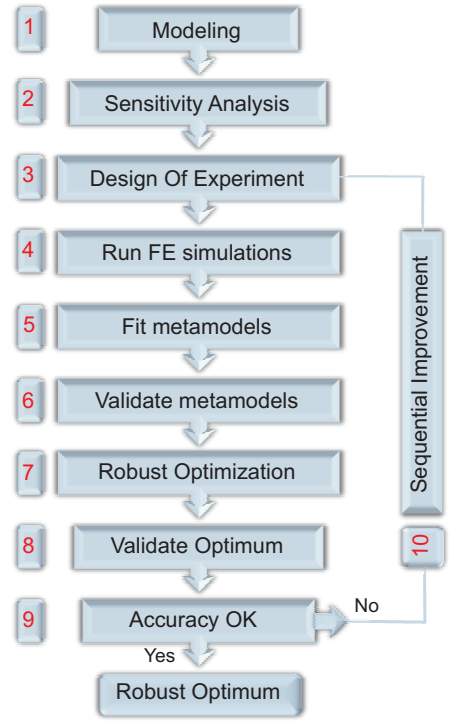

(b)

FIGURE 1. (a) Process-diagram and (b) flowchart of the robust optimization strategy

\section{MODELING AND SENSITIVITY ANALYSIS}

The first step (1) in the robust optimization strategy is to model the optimization problem under consideration. Both control and noise variables have to be selected and ranges have to be quantified. For the latter type of variables, a normal distribution is assumed. The stochastic variables can now be expressed by a mean value $\mu_{z}$ and a corresponding variance $\sigma_{z}^{2}$. The same holds for the objective function $f$ and constraints $\mathbf{g}$ since they can be influenced by the noise variables. Control variables $\mathbf{x}$ can be treated the same as in a deterministic problem where its ranges are bounded by Lower Bounds (lb) and Upper Bounds (ub). A typical variance-based robust optimization formulation is given by:

$$
\begin{aligned}
& \text { find } \mathbf{x} \\
& \begin{aligned}
\min & \mu_{f}+k_{f} \sigma_{f} \\
\text { s.t. } & \mathbf{L S L} \leq \mu_{\mathbf{g}} \pm \mathbf{k}_{\mathbf{g}} \sigma_{\mathbf{g}} \leq \mathbf{U S L} \\
& \quad \mathbf{l} \mathbf{b}_{x} \leq \mathbf{x} \leq \mathbf{u} \mathbf{b}_{x} \\
& \mathbf{z} \sim N\left(\mu_{z}, \sigma_{z}^{2}\right)
\end{aligned}
\end{aligned}
$$

In Equation 1, the objective function is to minimize the weighted sum of both the mean $\mu_{f}$ and standard deviation $\sigma_{f}$ of the response, i.e. both the location and the width of the response distribution are minimized simultaneously. A similar type of formulation is used for describing the constraints. Note that using the weighted sum formulation for a constraint can also be interpreted as a reliability constraint that ensures a $3 \sigma$ reliability with respect to a certain Lower Specification Limit (LSL) and Upper Specification Limit (USL) for $\mathrm{k}_{g}=3$. If $\mathbf{g}$ is assumed to be normally distributed, then one can subsequently calculate the scrap rate. 
Before proceeding with the actual robust optimization, a sensitivity analysis is performed in step (2). Screening techniques are applied to reduce the size and complexity of the optimization problem. This reduction is possible based on the Pareto effect stating that $80 \%$ of the consequences stem from $20 \%$ of the causes. In the context of optimization, screening techniques for variable reduction are used to determine the control and noise variables that have the most effect on the objective function and implicit constraint(s).

\section{ROBUST OPTIMIZATION AND VALIDATION}

Solving the robust optimization problem is performed in the following steps. A (3) Design Of Experiment (DOE) is performed based on a full factorial design combined with a space filling Latin Hypercube Design (LHD). After having run the FE simulations (4) corresponding to the settings specified by the DOE, a metamodel is fitted in the combined control-noise variable space using both Response Surface Methodology (RSM) and Kriging metamodeling techniques (5). In the robust optimization strategy, the computationally expensive non-linear FE simulations are thus replaced by a surrogate model.

Metamodel validation (6) is performed using ANalysis Of VAriance (ANOVA) techniques [4]. To estimate the performance of the approximate model, leave-one-out cross-validation is used. Each DOE point is selected once as the validation data, and the remaining DOE points as the training data. The level of fit of each metamodel is calculated and used to select the most accurate metamodel with respect to the FE-model response. From the single metamodel, two models for the response mean and variance are subsequently extracted using Monte Carlo Analyses (MCA) of 10.000 points each. The latter step can be performed very efficiently since the MCA is executed onto the metamodel. Both models of the mean and variance can now be used for robust optimization (7) by applying a Genetic Algorithm (GA).

Initially, a minimum number of DOE points is chosen in step (3) to limit the required number of expensive function evaluations. The design engineer must be aware of the fact that the solution of the approximated problem is only an estimate of the true robust optimum. To obtain an accurate and reliable solution, the metamodels have to be validated (8). This is done by performing approximately $100 \mathrm{FE}$ simulations in the robust optimum using Latin Hypercube Sampling (LHS). The resulting coarse estimation of the robustness criterion is used to validate the accuracy of the metamodel prediction (9). If the accuracy of the robustness prediction is not sufficient according to the design engineer, a sequential improvement step can be applied to update the metamodel successively (10).

\section{SEQUENTIAL ROBUST OPTIMIZATION}

The goal of sequential optimization is to increase the accuracy of the objective function prediction at regions of interest containing the optimal design. This is achieved by adding new DOE points to the original set. An update algorithm is required to select the location of the next infill point. The algorithm applied in this work will be outlined in the following subsections. After evaluating the new infill point, the metamodels are updated and validated taking into account the additional response. A robust optimization procedure is started and the new prediction of the robust optimum is determined. Instead of validating this optimum using FE simulations, a stopping criterion is evaluated. The sequential improvement strategy continues to add DOE points until the stopping criterion is fulfilled. As a final check, the design engineer can decide to validate the accuracy of the newly predicted robust optimum.

\section{Expected Improvement Criterion}

In $[5,6]$, an update algorithm is proposed based on the principle of Expected Improvement (EI). The proposed Efficient Global Optimization (EGO) strategy adds additional sample points by maximizing the EI. The Improvement is defined as:

$$
\mathrm{I}= \begin{cases}0 & \text { if } y(\mathbf{x})>f_{\min } \\ f_{\min }-y(\mathbf{x}) & \text { otherwise }\end{cases}
$$

The improvement is calculated by taking the difference between the objective function value $y(\mathbf{x})$ with respect to the minimum feasible sampled (or tested) response of the true objective function value $f_{\min }$ if $y(\mathbf{x})<f_{\min }$. In addition to a 
mean value prediction $\hat{y}\left(x_{0}\right)$ at an untried setting $x_{0}$, the EI algorithm makes use of the prediction error $\hat{s}\left(x_{0}\right)$ provided by the metamodel. In other words, the predictor $\hat{y}\left(x_{0}\right)$ represents a realization of a stochastic process $Y$ in which the randomness is governed by the uncertainty $\hat{s}\left(x_{0}\right)$ about the true objective function; see e.g. [7, 8]. The so-called posterior distribution at $x_{0}$ can be modeled as a normal distribution with $Y \sim N\left(\hat{y}\left(x_{0}\right), \hat{s}^{2}\left(x_{0}\right)\right)$.

Replacing $y(\mathbf{x})$ in Equation 2 by the random variable $Y$ will result in an expression of the improvement that is also random variable. As shown in [6], the expected value of the improvement can now be expressed in a closed form given by:

$$
\mathrm{E}(I)= \begin{cases}\left(f_{\min }-\hat{y}\right) \Phi\left(\frac{f_{\min }-\hat{y}}{\hat{s}}\right)+\hat{s} \phi\left(\frac{f_{\min }-\hat{y}}{\hat{s}}\right) & \text { if } \hat{s}>0 \\ 0 & \text { if } \hat{s}=0\end{cases}
$$

with $\phi$ and $\Phi$ the probability density and cumulative distribution function of the standard normal distribution respectively. For notational simplicity, the dependence on $\mathbf{x}$ is omitted here. The first term in Equation 3 contributes to the EI if $\hat{y}$ is smaller than $f_{\min }$. The second term contributes to the EI at locations of high uncertainty about whether $\hat{y}$ will be better than $f_{\text {min }}$. The criterion is thus capable of searching both locally (first term) and globally (second term). Maximizing the EI finally provides the coordinates of the infill point $\mathbf{x}^{\prime}$.

\section{Extension of the Expected Improvement Criterion}

In the case of robust sequential optimization, the goal is to find a suitable location of the infill point which requires the determination of an optimal setting for both the control variables $\mathbf{x}^{\prime}$ and noise variables $\mathbf{z}^{\prime}$. Focusing on the EI algorithm, several difficulties arise if noise variables are taken into account. Firstly, the goal is to find an infill point that is most promising with respect to the robustness criterion instead of the metamodel itself. Secondly, the prediction uncertainty or suitable error estimation $\hat{s}$ associated with respect to the objective function is not accounted for.

Only a few studies have been published that consider sequential update strategies in which noise variables are taken into account; see e.g. [9, 10,11]. The robust sequential optimization algorithm that is applied in this work is proposed in [12]. The search for an infill point in the control-noise space is divided into two steps. First, $\mathbf{x}^{\prime}$ is determined in the control space after which $\mathbf{z}^{\prime}$ is identified in the noise space. Determining $\mathbf{x}^{\prime}$ requires the evaluation of the robustness criterion by application of a MCA onto the metamodel. As a result, the majority of the sampling points that are incorporated in the prediction are untested. That means that an uncertainty $\hat{s}$ about the predictor $\hat{y}$ at each point $\left(\mathbf{x}, \mathbf{z}_{i}\right)$ remains present. As a result, two random variables are obtained in the same probability space. Each point $\left(\mathbf{x}, \mathbf{z}_{i}\right)$ is now a realization of the stochastic noise variable $Z$ and the posterior error distribution in this point. The stochastic process $Y$ can now be written as $(Y \mid Z) \sim N\left(\hat{y}(Z), \hat{s}^{2}(Z)\right)$. To evaluate the prediction uncertainty with respect to the robustness criterion, the conditional variance formula is used:

$$
\operatorname{var}(Y)=\mathrm{E}\left(\hat{s}(Z)^{2}\right)+\sigma_{Y}^{2}
$$

The presence of prediction uncertainty adds an additional term to the prediction variance of the stochastic process $Y$ which equals the expected value of $\hat{s}(Z)^{2}$ over the noise space. Returning back to the definition of the EI, a revision has to be done. This is because the definition of the current best solution of the robustness criterion can now also be seen as a random process with $\left(Y^{*} \mid Z\right) \sim N\left(\hat{y}^{*}(Z), \hat{s}^{*}(Z)^{2}\right)$. The EI can now be written as:

$$
\mathrm{E}(I)=\int_{a}^{b}\left(\hat{y}^{*}-y\right)\left(f_{Y}(y)-f_{Y^{*}}(y)\right) d y
$$

with $f_{Y}(y)$ and $f_{Y^{*}}(y)$ the probability density function of the stochastic process $Y$ and current best solution $Y^{*}$. The integration bounds $a$ and $b$ depend on the intersection points of $f_{Y}(y)$ and $f_{Y^{*}}(y)$ which can be calculated analytically. As a first step, the current best solution is calculated by evaluating the robustness criterion for all settings of the control variable $\mathbf{x}=\mathbf{x}_{l}$ that are part of the set of DOE points. Subsequently, the EI can be maximized by evaluating Equation 5 for a grid of points $\mathbf{x}$ representing the candidate infill points. In exploring the noise space, the deteriorating effects of the noise variables are of special interest. Maximizing the product of the error prediction and the probability of occurrence results in the optimal noise variable setting, i.e. $\mathbf{z}^{\prime}=\arg \max \left(\hat{s}\left(\mathbf{x}^{\prime}, \mathbf{z}\right)^{2} f_{Z}(\mathbf{z})\right)$. Together, these optimal settings $\left(\mathbf{x}^{\prime}, \mathbf{z}^{\prime}\right)$ define the infill point at which a new FE simulation is performed. The resulting FE response is added to the set of responses and the metamodel is updated. The update sequence continues until a threshold value for the EI or a maximum number of runs is reached. 


\section{APPLICATION TO A V-BENDING PROCESS}

The proposed new robust optimization strategy will now be applied to optimize an industrial V-bending process. The goal of this optimization study is to gain more insight into the production process. With that, the process can be optimized such that products are produced within specification in a robust way. Figure 2(a) shows the 2D FE-model of half of the part, MSC Marc has been used as FE-code. One simulation takes about 7 minutes to perform. Both the die and punch are modeled to be non-rigid such that the real process is represented more realistically.

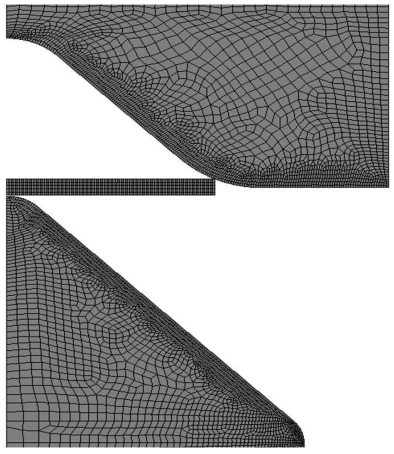

(a)

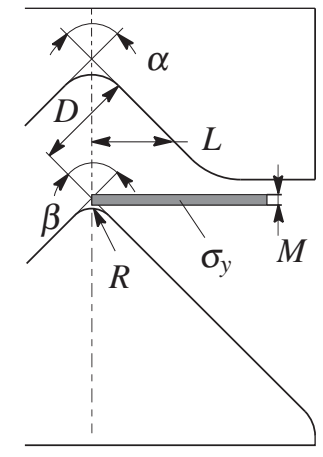

(b)

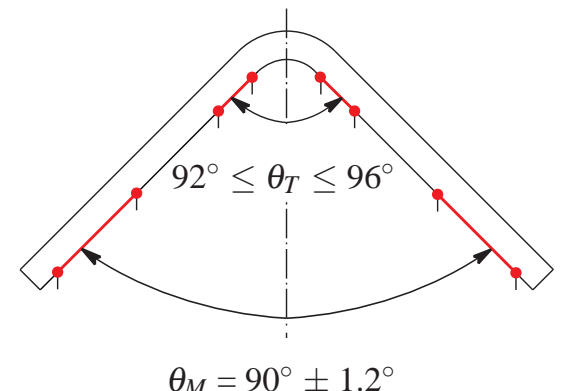

(c)

FIGURE 2. (a) 2D FE-model, (b) definition of control and noise variables and (c) constraints on the flange shape

\section{Modeling and Robust Optimization}

A sensitivity analysis is first performed to reduce the size and complexity of the optimization problem. As a result, the number of variables has been reduced to four control variables and two noise variables. The remaining set of variables is shown in Figure 2(b). The control variables are the radius of the die $(R)$, the final distance $(D)$ between the flange of the die and punch (if no deformation of the tooling would occur), the dimensions of the punch $(L)$ and the angle of the die $(\alpha)$ and the punch $(\beta)$. Since $\alpha$ and $\beta$ must be equal, both angles can be described by a single control variable. As with the process in practice, the material thickness $(M)$ and yield stress $\left(\sigma_{y}\right)$ are considered uncertain. To ensure a correct performance of the final product, constraints on the flange shape of the product are prescribed. The flange shape is defined by a transition angle $\left(\theta_{T}\right)$ and a main angle $\left(\theta_{M}\right)$ spanned up by the marked line segments; see Figure 2(c). The constraint on the main angle is stricter since this angle is most critical with respect to the performance of the final product. In the current V-bending process, active steering of $D$ is required to obtain products that satisfy the requirements for both angles. The goal of the robust optimization study is to achieve an $3 \sigma$ process for both angles without the need to adjust $D$. The main angle is taken into account as the objective function $f$ while satisfying $\pm 3 \sigma$ constraints on the transition angle:

$$
\begin{array}{cc}
\min & \left|\left(\mu_{\theta_{M}}-90\right)\right|+3 \sigma_{\theta_{M}} \\
\text { s.t. } & 22 \leq \mu_{\theta_{T}}-3 \sigma_{\theta_{T}} \\
& \mu_{\theta_{T}}+3 \sigma_{\theta_{T}} \leq 96 \\
& 92 \leq \alpha=\beta \leq 93 \\
1 & \leq R \leq 1.3 \\
& 4 \leq L \leq 5 \\
& 0.4 \leq D \leq 0.65 \\
& M \sim N\left(0.51,0.01^{2}\right) \\
& \sigma_{y} \sim N\left(350,6.66^{2}\right)
\end{array}
$$




\section{Results and Evaluation}

As a basis for the metamodel, an initial DOE of 300 points is constructed in the combined 6D control-noise variable space. The FE simulations are performed on four parallel processors reducing the total calculation time to approximately 9 hours so that all simulations can be performed overnight. Using ANOVA, a second-order Kriging model resulted in the most accurate fit for both $f$ and $g$. Impressions of the metamodels are shown in Figure 3.

Figure 3(a) and 3(b) show the metamodels of the main angle as a function of the distance $D$, and the material thickness $M$ and yield stress $\sigma_{y}$ respectively. For visualization purposes, the remaining variables are set to their current process settings as shown in the first column of Table 1. Evaluating the shape of the metamodel, an increase of the main angle is observed for large $D$ since the material is only bent and not yet flattened. Note that for small $D$, the main angle approaches the tooling angle caused by the flattening of the material. In between these extreme settings, an area can be observed in which both the influence of the distance $D$ and both noise variables is small. For this specific setting, this could potentially yield a process which is robust with respect to noise. Moreover, by looking at the slope of the metamodel in the noise direction it can be seen that the standard deviation increases for increasing $D$. A similar behavior is observed for the transition angle; see Figures 3(c) and 3(d). Note that especially the lower constraint on the transition angle (presented by the lower plane) significantly decreases the feasible area also excluding the robust area.

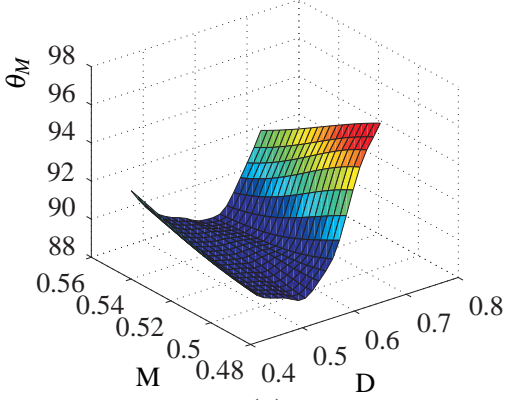

(a)

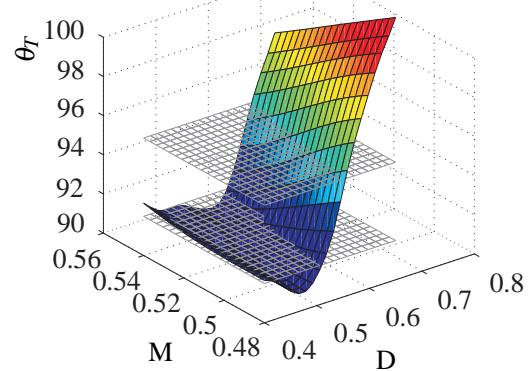

(c)

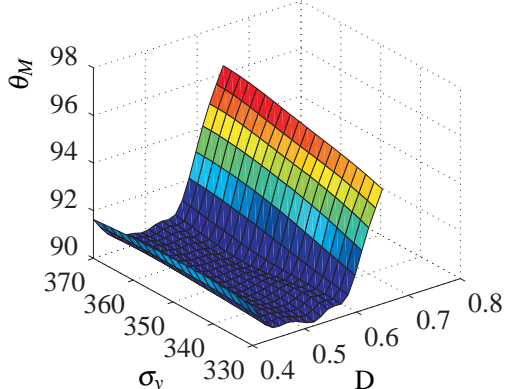

(b)

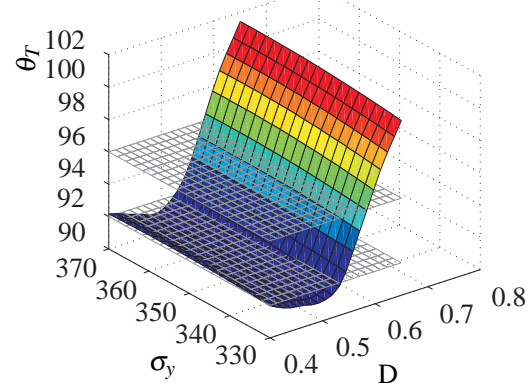

(d)

FIGURE 3. Kriging metamodel impressions of the (a,b) main and (c,d) transition angle

The metamodels are subsequently used for optimization, solving Formulation (6). The initial optimization approach did not result in a $3 \sigma$ process. This is mainly caused by a non-robust behavior of the transition angle in the feasible design space. Therefore, the $3 \sigma$ constraint on the transition angle is slightly relaxed to a $2 \sigma$ constraint. The optimal process settings for the revised optimization problem are given in the second column of Table 1 . The results of the optimized process are shown in Figure 4 in which the main and transition angle are plotted as a function of $D$. The vertical bars represent the $\pm 3 \sigma$ bounds caused by the influence of the noise variables. The optimal distance $D$ is found to be $0.55 \mathrm{~mm}$ which corresponds to a mean value of the main angle of $90.39^{\circ}$ and a standard deviation of $0.1^{\circ}$; see Table 2. Note that the modified $2 \sigma$ requirement for the moment matching constraint on $g$ has been satisfied.

In the case of deterministic optimization, neglecting the influence of the noise variables will result in an optimum that lies at the boundary of the lower constraint on $\theta_{T}$ for $D=0.52 \mathrm{~mm}$. The variation in $M$ and $\sigma_{y}$ will in this case lead to a high number of violations of the constraint in the real V-bending process. From this, it can be concluded that the effect of adding noise is significant if one compares the deterministic to the robust optimal settings for $D$. 
TABLE 1. Current process settings, process settings after robust optimization and after sequential robust optimization

\begin{tabular}{cccc}
\hline Parameter & Current Settings & Robust Optimization & Sequential Robust Optimization \\
\hline$\alpha, \beta$ & $92^{\circ}$ & $92.4^{\circ}$ & $92.9^{\circ}$ \\
$R$ & $1.15 \mathrm{~mm}$ & $1.16 \mathrm{~mm}$ & $1.14 \mathrm{~mm}$ \\
$L$ & $5 \mathrm{~mm}$ & $4.8 \mathrm{~mm}$ & $4.9 \mathrm{~mm}$ \\
$D$ & adaptive & $0.55 \mathrm{~mm}$ & $0.55 \mathrm{~mm}$ \\
\hline
\end{tabular}

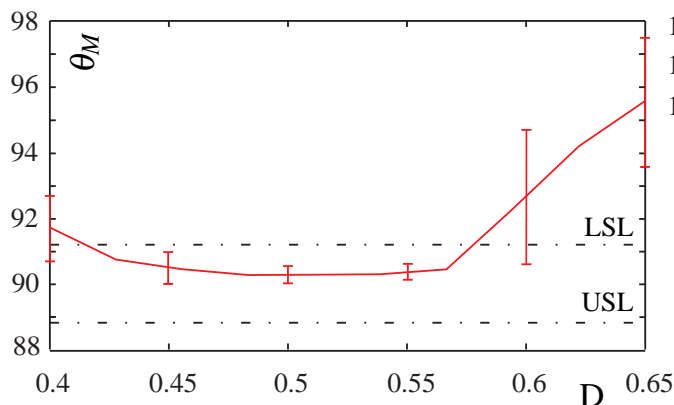

(a)

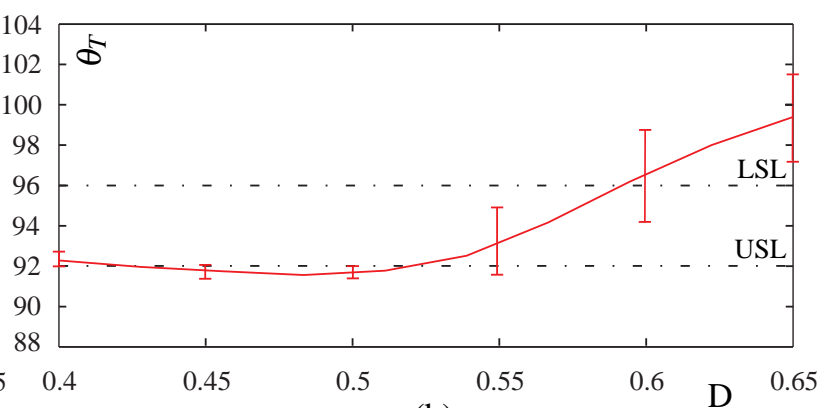

(b)

FIGURE 4. Optimal process results for the (a) main and (b) transition angle as a function of the distance $D$

\section{Validation and Sequential Robust Optimization}

The prediction accuracy in the robust optimum is evaluated by a first validation step from which the results are presented in Table 2. The FE simulation based prediction of the mean value of both the objective function and the constraint show a deviation in comparison to the metamodel based predictions. Next to that, a small increase of the standard deviation is obtained for both responses. From this it can be concluded that the initial metamodels approximate the standard deviation rather well. The prediction of the mean value in the vicinity or the robust optimum is less accurate which leaves room for sequential optimization. It must be noted here that drawing firm conclusions about the exact prediction accuracy is risky due to the limited number of FE simulations. However, further increasing the number of expensive function evaluations would decrease the efficiency of the strategy. A trade-off has to be found. Using $100 \mathrm{FE}$ simulations in the validation step will provide the design engineer with a feeling of the approximation accuracy of the metamodel in the robust optimum.

TABLE 2. Response of the main and transition angle in the robust optimum

\begin{tabular}{lccccc}
\hline & \multicolumn{2}{c}{ First Validation Step } & & \multicolumn{2}{c}{ Second Validation Step } \\
\cline { 2 - 3 } \cline { 5 - 6 } Response type & Metamodel & FE simulations & & Metamodel & FE simulations \\
\hline$\mu_{\theta_{M}}$ & 90.39 & 90.69 & & 90.47 & 90.53 \\
$\sigma_{\theta_{M}}$ & 0.10 & 0.13 & & 0.10 & 0.12 \\
$\mu_{\theta_{M}}+3 \sigma_{\theta_{M}}$ & 90.69 & 91.08 & & 90.77 & 90.89 \\
$\mu_{\theta_{T}}$ & 93.33 & 93.77 & & 93.77 & 93.83 \\
$\sigma_{\theta_{T}}$ & 0.66 & 0.78 & & 0.85 & 0.82 \\
$\mu_{\theta_{T}}+2 \sigma_{\theta_{T}}$ & 94.65 & 95.33 & & 95.47 & 95.47 \\
$\mu_{\theta_{T}}-2 \sigma_{\theta_{T}}$ & 92.01 & 92.21 & & 92.07 & 92.19 \\
\hline
\end{tabular}

Executing the sequential improvement procedure resulted in an additional 32 DOE points, mainly clustering around the current robust optimum for varying tooling angles $\alpha$ and $\beta$. The settings of the robust optimum after sequential optimization are given in the third column of Table 1. The optimal setting of the tooling angle has increased where the other three control variables show negligible changes. Visualization of the metamodels shows that the location of the constrained robust optimum with respect to the tooling angle is highly sensitive for small changes in the shape of the constraint function. The combination of this phenomenon with an increased prediction accuracy of the constraint explains the shift of the robust optimum. 
The results of the second validation step are given in Table 2. The metamodel based and FE simulation based responses show an increase of the prediction accuracy of both the objective function and constraint in comparison to the first validation step. This shows that the sequential optimization step is an efficient way to increase the reliability of the robustness measure in the vicinity of the robust optimum.

Application of the robust optimization strategy to the V-bending process resulted in a significant improvement of the robustness $(>2 \sigma)$. This is achieved by improving the process design for robustness. The deteriorating effects of the noise variables are minimized by changing the tooling angle and determining the optimal process depth setting $(D)$. This will make the active steering of the depth setting in the current process redundant. Finally, valuable process insights are obtained, among others by visualization of the metamodels used in the robust optimization strategy.

\section{CONCLUSIONS AND FUTURE WORK}

The robust optimization strategy presented in this paper allows for modeling and solving robust optimization problems in the metal forming industries. Uncertainties such as material variation and process settings, are taken into account explicitly. The sequential update algorithm is based on an expected improvement measure and has proven to increase the accuracy of the objective function prediction at regions of interest in an efficient way. The developed strategy goes beyond just using deterministic strategies and algorithms, it assists metal forming industries to achieve robust and reliable mass production processes.

The challenge remains to balance the number of FE simulations spent on the robustness evaluation and the reliability of the robustness measurements themselves. Performing the validation step that is incorporated in the robust optimization strategy, is therefore absolutely mandatory. Focusing on the prediction accuracy of the initial metamodel in the V-bending study, the first validation step pointed out that the response prediction was quite accurate. This suggests that there is room for decreasing the number of initial DOE points. Locally increasing the prediction accuracy of the initial metamodel can subsequently be done by applying the sequential improvement algorithm. This will automatically increase the efficiency of the robust optimization strategy. More generally, future work will focus on including manufacturing variability, process robustness and reliability during optimization in an efficient manner.

\section{ACKNOWLEDGMENTS}

This research was carried out under the project number M22.1.08303 in the framework of the Research Program of the Materials innovation institute (www.m2i.nl).

\section{REFERENCES}

1. H.-G. Beyer and B. Sendhoff. Robust optimization - A comprehensive survey. Journal of Computer Methods in Applied Mechanics and Engineering, 196:3190-3218, 2007.

2. G.-J. Park, T.-H. Lee, K.-H. Lee, and K.-H. Hwang. Robust design: an overview. Journal of the American Institute of Aeronautics and Astronautics, 44:181-191, 2006.

3. M. H. A. Bonte. Optimisation strategies for metal forming processes. Phd thesis, University of Twente, 2007.

4. R. H. Myers and D. C. Montgomery. Response Surface Methodology. John Wiley \& sons, 2002. ISBN 0-471-41255-4.

5. M. Schonlau. Computer Experiments and Global Optimization. Phd thesis, University of Waterloo, 1997.

6. D.R. Jones, M. Schonlau, and Welch W.J. Efficient global optimization of expensive black-box functions. Journal of Global Optimization, 13:455-492, 1998.

7. J. Sacks, W.J. Welch, T.J. Mitchell, and H.P. Wynn. Design and analysis of computer experiments. Statistical Science, 4: 409-423, 1989. ISSN 0883-4237.

8. T.J. Santner, B.J. Williams, and W.I. Notz. The Design and Analysis of Computer Experiments. Springer Verlag, 2003. ISBN 0-387-95420-1.

9. B.J. Williams, T.J. Santner, and W.I. Notz. Sequential design of computer experiments to minimize integrated response functions. Statistica Sinica, 10:1133-1152, 2000.

10. D. Huang. Experimental Planning and Sequential Kriging Optimization using Variable Fidelity Data. Phd thesis, Ohio State University, 2005.

11. J. S. Lehman. Sequential Design of Computer Experiments for Robust Parameter Design. Phd thesis, Ohio State University, 2002.

12. F. Jurecka. Robust Design Optimization Based on Metamodeling Techniques. Phd thesis, Technischen Universität München, 2007. 